Pacific

Journal of

Mathematics

COMPACTNESS AND THE PALAIS-SMALE PROPERTY FOR CRITICAL KIRCHHOFF EQUATIONS IN CLOSED MANIFOLDS

EMMANUEL HEBEY 


\title{
COMPACTNESS AND THE PALAIS-SMALE PROPERTY FOR CRITICAL KIRCHHOFF EQUATIONS IN CLOSED MANIFOLDS
}

\author{
EMMANUEL HEBEY
}

\begin{abstract}
We prove the Palais-Smale property and the compactness of solutions for critical Kirchhoff equations using solely energy arguments in the situation where no sign assumption is made on the solutions. We then prove the existence of a mountain-pass solution to the equation, discuss its ground-states structure, and, in extreme cases, prove uniqueness of this solution.
\end{abstract}

The Kirchhoff equation [1883] was proposed as an extension of the classical wave equation of D'Alembert for the vibration of elastic strings. The model takes into account the small vertical vibrations of a stretched elastic string when the tension is variable but the ends of the string are fixed. The equation in [loc. cit.] was written as

$$
\rho \frac{\partial^{2} u}{\partial t^{2}}-\left(\frac{P_{0}}{h}+\frac{E}{2 L} \int_{0}^{L}\left|\frac{\partial u}{\partial x}\right|^{2} d x\right) \frac{\partial^{2} u}{\partial x^{2}}=0,
$$

where $L$ is the length of the string, $h$ is the area of the cross-section, $E$ is the young modulus of the material (also referred to as the elastic modulus - it measures the string's resistance to being deformed elastically), $\rho$ is the mass density, and $P_{0}$ is the initial tension. Almost one century later, Jacques-Louis Lions [1978] returned to the equation and proposed a general Kirchhoff equation in arbitrary dimension with external force term which was written as

where

$$
\frac{\partial^{2} u}{\partial t^{2}}+\left(a+b \int_{\Omega}|\nabla u|^{2} d x\right) \Delta u=f(x, u)
$$

$$
\Delta=-\sum \frac{\partial^{2}}{\partial x_{i}^{2}}
$$

is the Laplace-Beltrami Euclidean Laplacian. We investigate in this paper the stationary version of this equation, in the case of closed manifolds, and when $f$ is the critical pure power nonlinearity. We prove the surprising result that the equation satisfies the Palais-Smale property when $a$ and $b$ are large (in a sense to be made

MSC2010: 58J05.

Keywords: compactness, ground-states, Kirchhoff equation, mountain-pass solution, Palais-Smale property. 
precised in Theorem 1 below). As usual, solutions of the stationary equation (with the square of the phase added as a potential) correspond to standing wave solutions of the evolution equation.

In what follows, we let $(M, g)$ be a closed $n$-dimensional Riemannian manifold of dimension $n \geq 4, a, b>0$ be positive real numbers, and $h \in C^{1}(M, \mathbb{R})$. The Kirchhoff equation we investigate is written as

$$
\left(a+b \int_{M}|\nabla u|^{2} d v_{g}\right) \Delta_{g} u+h u=|u|^{2^{\star}-2} u,
$$

where $2^{\star}=2 n /(n-2)$ is the critical Sobolev exponent. It is an appealing mathematical model because of its nonlocal nature and its integrodifferential structure. It has been paid much attention over the past years. Among other possible references (the following list is far from being exhaustive), we mention Figueiredo [2013], Figueiredo, Ikoma, and Santos [Figueiredo et al. 2014], Figueiredo and Santos [2012], He and Zou [2012], and the references in these papers. The case of positive solutions in the curved setting of closed manifolds has been investigated in Hebey and Thizy [2015a; 2015b]. We treat here the case where absolutely no sign assumption is made on the solutions. As a remark, the equation always has a pair of constant solutions if $h>0$ is constant.

In what follows, we let $H^{1}$ be the Sobolev space of functions in $L^{2}$ with one derivative in $L^{2}$. We let also $I: H^{1} \rightarrow \mathbb{R}$ be the functional

$$
I(u)=\frac{a}{2} \int_{M}|\nabla u|^{2} d v_{g}+\frac{b}{4}\left(\int_{M}|\nabla u|^{2} d v_{g}\right)^{2}+\frac{1}{2} \int_{M} h u^{2} d v_{g}-\frac{1}{2^{\star}} \int_{M}|u|^{2^{\star}} d v_{g} .
$$

As is easily checked, critical points of $I$ are solutions of (1). In particular, (1) has a variational structure. A sequence $\left(u_{\alpha}\right)_{\alpha}$ in $H^{1}$ is said to be a Palais-Smale sequence for $I$ if the sequence $\left(I\left(u_{\alpha}\right)\right)_{\alpha}$ is bounded with respect to $\alpha$, and $I^{\prime}\left(u_{\alpha}\right) \rightarrow 0$ in $\left(H^{1}\right)^{\prime}$ as $\alpha \rightarrow+\infty$. Following standard terminology, we say that $I$ satisfies the PalaisSmale property if Palais-Smale sequences for $I$ converge, up to a subsequence, in $H^{1}$. Let $S_{n}$ be the sharp Euclidean Sobolev constant given by $S_{n}=\frac{1}{4} n(n-2) \omega_{n}^{2 / n}$, where $\omega_{n}$ is the volume of the unity $n$-sphere. We define the dimensional constant $C(n)$ by

$$
C(n)=\frac{2(n-4)^{(n-4) / 2}}{(n-2)^{(n-2) / 2} S_{n}^{n / 2}} .
$$

The main result of this paper provides very simple criteria on $a$ and $b$ for the equation to be compact and $I$ to satisfy the Palais-Smale property. Our main result is stated as follows.

Theorem 1. Suppose that $(M, g)$ is a closed n-dimensional Riemannian manifold of dimension $n \geq 4$, that $a, b>0$ are positive real numbers, and that $h \in C^{1}(M, \mathbb{R})$ makes $\Delta_{g}+h / a$ positive. Assume that $b \gg 1$ when $n=4$, and that $a^{(n-4) / 2} b>C(n)$ 
when $n \geq 5$, where $C(n)$ is as in (3). Then, I satisfies the Palais-Smale property and the set of solutions of (1) is compact in the $C^{2}$-topology.

It is very surprising that such a compactness result, in strong topologies, for an equation with critical nonlinearity, can be obtained without the whole machinery of strong pointwise estimates (see Hebey [2014] for a reference in book form on this machinery). Moreover, no assumption of positiveness is made on the solutions in Theorem 1.

Proof of Theorem 1. (i) We prove that Palais-Smale sequences for $I$ are bounded in $H^{1}$, assuming that $b \gg 1$ when $n=4$. Let $\left(u_{\alpha}\right)_{\alpha}$ be a Palais-Smale sequence for $I$. Then, we get that $I\left(u_{\alpha}\right)=O(1)$ and $I^{\prime}\left(u_{\alpha}\right) \cdot\left(u_{\alpha}\right)=o\left(\left\|u_{\alpha}\right\|_{H^{1}}\right)$, where $\|\cdot\|_{H^{1}}$ is the $H^{1}$-norm given for $u \in H^{1}$ by

$$
\|u\|_{H^{1}}^{2}=\|\nabla u\|_{L^{2}}^{2}+\|u\|_{L^{2}}^{2} .
$$

In particular,

$$
a \int_{M}\left(\left|\nabla u_{\alpha}\right|^{2}+\frac{h}{a} u_{\alpha}^{2}\right) d v_{g}+b\left(\int_{M}\left|\nabla u_{\alpha}\right|^{2} d v_{g}\right)^{2}=\int_{M}\left|u_{\alpha}\right|^{2^{\star}} d v_{g}+o\left(\left\|u_{\alpha}\right\|_{H^{1}}\right)
$$

and that

$$
\frac{a}{2} \int_{M}\left(\left|\nabla u_{\alpha}\right|^{2}+\frac{h}{a} u_{\alpha}^{2}\right) d v_{g}+\frac{b}{4}\left(\int_{M}\left|\nabla u_{\alpha}\right|^{2} d v_{g}\right)^{2}=\frac{1}{2^{\star}} \int_{M}\left|u_{\alpha}\right|^{2^{\star}} d v_{g}+O(1) .
$$

By the Sobolev-Poincaré inequality, there exist $C_{1}, C_{2}>0$ such that

$$
\left\|u_{\alpha}\right\|_{L^{2^{\star}}}^{2^{\star}} \leq C_{1}\left\|\nabla u_{\alpha}\right\|_{L^{2}}^{2^{\star}}+C_{2}\left|\bar{u}_{\alpha}\right|^{2^{\star}}
$$

for all $\alpha$, where

$$
\bar{u}_{\alpha}=\frac{1}{V_{g}} \int_{M} u_{\alpha} d v_{g}
$$

is the average of $u_{\alpha}$, and by the Poincaré inequality,

$$
\left\|u_{\alpha}-\bar{u}_{\alpha}\right\|_{L^{2}}^{2} \leq \frac{1}{\lambda_{1}}\left\|\nabla u_{\alpha}\right\|_{L^{2}}^{2}
$$

for all $\alpha$, where $\lambda_{1}=\lambda_{1}(M, g)>0$ is the first nonzero eigenvalue of $\Delta_{g}$. It clearly follows from the positivity of $\Delta_{g}+h / a$, (5), and (6) that if either $n=4$ and $b>C_{1}$ or $n \geq 5$ and if $\bar{u}_{\alpha}=O(1)$, then $\left\|u_{\alpha}\right\|_{H^{1}}=O(1)$. We may therefore assume that $\bar{u}_{\alpha} \rightarrow+\infty$ as $\alpha \rightarrow+\infty$. Then, still by the positivity of $\Delta_{g}+h / a$, (5), and (6),

$$
\int_{M}\left|\nabla u_{\alpha}\right|^{2} d v_{g}= \begin{cases}\frac{1}{b} O\left(\bar{u}_{\alpha}^{2}\right) & \text { if } n=4, \\ o\left(\bar{u}_{\alpha}^{2}\right) & \text { if } n \geq 5,\end{cases}
$$

where we assume that $b>C_{1}$ when $n=4$. Now, we write that

$$
u_{\alpha}=\bar{u}_{\alpha}\left(1+\varphi_{\alpha}\right) \text {. }
$$


Then, $\int_{M} \varphi_{\alpha} d v_{g}=0$ and

$$
\bar{u}_{\alpha}^{2} \int_{M}\left|\nabla \varphi_{\alpha}\right|^{2} d v_{g}=\int_{M}\left|\nabla u_{\alpha}\right|^{2} d v_{g} .
$$

It follows from (8), (10), the Poincaré inequality, (7), and (10) that

$$
\left\|\varphi_{\alpha}\right\|_{H^{1}}^{2}= \begin{cases}O\left(\frac{1}{b}\right) & \text { if } n=4 \\ o(1) & \text { if } n \geq 5 .\end{cases}
$$

In particular, by (9) and (11),

$$
\int_{M}\left(\left|\nabla u_{\alpha}\right|^{2}+\frac{h}{a} u_{\alpha}^{2}\right) d v_{g}=\bar{u}_{\alpha}^{2}\left(1+A_{\alpha}\right) \quad \text { and } \quad \int_{M}\left|u_{\alpha}\right|^{2^{\star}} d v_{g}=\bar{u}_{\alpha}^{2^{\star}}\left(1+B_{\alpha}\right),
$$

where $A_{\alpha}=O\left(\frac{1}{b}\right)$ and $B_{\alpha}=O\left(\frac{1}{b}\right)$ if $n=4$, and $A_{\alpha}=o(1)$ and $B_{\alpha}=o(1)$ if $n \geq 5$. Subtracting $\frac{1}{4}$ of (4) from (5) yields

$$
\frac{a}{4} \int_{M}\left(\left|\nabla u_{\alpha}\right|^{2}+\frac{h}{a} u_{\alpha}^{2}\right) d v_{g}=\left(\frac{1}{2^{\star}}-\frac{1}{4}\right) \int_{M}\left|u_{\alpha}\right|^{2^{\star}} d v_{g}+O(1)+O\left(\left\|u_{\alpha}\right\|_{H^{1}}\right) .
$$

Picking $b \gg 1$ when $n=4$, the contradiction follows by combining (12) and (13). This proves that $\left(u_{\alpha}\right)_{\alpha}$ is bounded in $H^{1}$.

(ii) We prove that $I$ satisfies the Palais-Smale property assuming that $b \gg 1$ when $n=4$, and that $a^{(n-4) / 2} b>C(n)$ when $n \geq 5$. We let

$$
K_{\alpha}=a+b \int_{M}\left|\nabla u_{\alpha}\right|^{2} d v_{g},
$$

$h_{\alpha}=K_{\alpha}^{-1} h$, and

$$
v_{\alpha}=\left(\frac{1}{K_{\alpha}}\right)^{\frac{1}{2^{\star}-2}} u_{\alpha} .
$$

We define $I_{\alpha}: H^{1} \rightarrow \mathbb{R}$ by

$$
I_{\alpha}(u)=\frac{1}{2} \int_{M}\left(|\nabla u|^{2}+h_{\alpha} u^{2}\right) d v_{g}-\frac{1}{2^{\star}} \int_{M}|u|^{2^{\star}} d v_{g} .
$$

According to (i), and up to passing to a subsequence, $K_{\alpha} \rightarrow K_{\infty}$ as $\alpha \rightarrow+\infty$ for some $K_{\infty} \geq a$. In particular, $\left(h_{\alpha}\right)_{\alpha}$ converges in $C^{k}$ for all $k$, and $\left(v_{\alpha}\right)_{\alpha}$ is bounded in $H^{1}$. This implies that $I_{\alpha}\left(v_{\alpha}\right)=O(1)$, and, as one can check,

$$
I_{\alpha}^{\prime}\left(v_{\alpha}\right) \cdot(\varphi)=\left(\frac{1}{K_{\alpha}}\right)^{\frac{2^{\star}-1}{2^{\star}-2}} I^{\prime}\left(u_{\alpha}\right) \cdot(\varphi)
$$

for all $\varphi \in H^{1}$. Then $\left(v_{\alpha}\right)_{\alpha}$ is a Palais-Smale sequence for the family $\left(I_{\alpha}\right)_{\alpha}$ (in the sense of Hebey [2014]). In particular the $H^{1}$-decomposition as in Struwe [1984] applies (see Druet, Hebey, and Robert [Druet et al. 2004], Hebey [2014], and Vétois 
[2007] for the closed setting with varying potentials), and we get that there exists $v_{\infty} \in H^{1}, k \in \mathbb{N}$, and $k+1$ sequences $\left(B_{1, \alpha}\right)_{\alpha}, \ldots,\left(B_{k, \alpha}\right)_{\alpha},\left(R_{\alpha}\right)_{\alpha}$ in $H^{1}$ such that

$$
v_{\alpha}=v_{\infty}+\sum_{i=1}^{k} B_{i, \alpha}+R_{\alpha} \text { in } M
$$

and

$$
\int_{M}\left|\nabla v_{\alpha}\right|^{2} d v_{g}=\int_{M}\left|\nabla v_{\infty}\right|^{2} d v_{g}+\sum_{i=1}^{k} \int_{M}\left|\nabla B_{i, \alpha}\right|^{2} d v_{g}+o(1)
$$

for all $\alpha, R_{\alpha} \rightarrow 0$ in $H^{1}$ as $\alpha \rightarrow+\infty$ and the "bubbles" $\left(B_{i, \alpha}\right)_{\alpha}$ satisfy the following properties for any $i=1, \ldots, k$ :

(a) $B_{i, \alpha} \rightarrow 0$ in $L^{2}$ as $\alpha \rightarrow+\infty$,

(b) $\left\|B_{i, \alpha}\right\|=O(1)$, and

(c) $\int_{M}\left|\nabla B_{i, \alpha}\right|^{2} d v_{g} \geq S_{n}^{n / 2}+o(1)$ for all $\alpha$,

where $S_{n}$ is the sharp Euclidean constant as in (3). In (c), there is equality if each $B_{i, \alpha}$ is positive. Then, since $\left(u_{\alpha}\right)_{\alpha}$ is bounded in $H^{1}$, and by (17)-(18), we get that, up to passing to a subsequence,

$$
\begin{aligned}
K_{\alpha} & =a+b \int_{M}\left|\nabla u_{\alpha}\right|^{2} d v_{g} \\
& =a+b K_{\alpha}^{2 /\left(2^{\star}-2\right)} \int_{M}\left|\nabla v_{\alpha}\right|^{2} d v_{g} \\
& =a+b K_{\alpha}^{2 /\left(2^{\star}-2\right)} \int_{M}\left|\nabla v_{\infty}\right|^{2} d v_{g}+b K_{\alpha}^{2 /\left(2^{\star}-2\right)} \sum_{i=1}^{k} \int_{M}\left|\nabla B_{i, \alpha}\right|^{2} d v_{g}+o(1) \\
& =a+b K_{\infty}^{2 /\left(2^{\star}-2\right)} \int_{M}\left|\nabla v_{\infty}\right|^{2} d v_{g}+b C K_{\infty}^{2 /\left(2^{\star}-2\right)}+o(1),
\end{aligned}
$$

where $C \geq k S_{n}^{n / 2}$. In particular, by (19),

$$
K_{\infty}=a+b K_{\infty}^{2 /\left(2^{\star}-2\right)} \int_{M}\left|\nabla v_{\infty}\right|^{2} d v_{g}+b C K_{\infty}^{2 /\left(2^{\star}-2\right)} .
$$

When $n=4$, we have $2 /\left(2^{\star}-2\right)=1$, and (20) implies that $k=0$ in (17) as soon as $b \gg 1$. In particular, the sequence $\left(u_{\alpha}\right)_{\alpha}$ converges strongly in $H^{1}$, and $I$ satisfies the Palais-Smale property. When $n \geq 5$, we define

$$
f(x)=b k S_{n}^{n / 2} x^{(n-2) / 2}-x+a .
$$


By (20), and since $C \geq k S_{n}^{n / 2}$, we have that $f\left(K_{\infty}\right) \leq 0$. Assuming that $k \geq 1$, noting that $f$ is minimum at $x_{0}$, where

we compute that

$$
x_{0}=\left(\frac{2}{(n-2) b k S_{n}^{n / 2}}\right)^{2 /(n-4)},
$$

$$
f\left(x_{0}\right)=-\frac{n-4}{n-2}\left(b k S_{n}^{n / 2}\right)^{-2 /(n-4)}\left(\frac{2}{n-2}\right)^{2 /(n-4)}+a .
$$

If $f\left(K_{\infty}\right) \leq 0$, then $f\left(x_{0}\right) \leq 0$, and by $(21), b k a^{(n-4) / 2} \leq C(n)$. Since by assumption $a^{(n-4) / 2} b>C(n)$, it must be the case that $k=0$ in (17). In particular, the sequence $\left(u_{\alpha}\right)_{\alpha}$ converges strongly in $H^{1}$, and $I$ satisfies the Palais-Smale property also when $n \geq 5$.

(iii) We prove the compactness of (1) assuming that $b \gg 1$ when $n=4$ and that $a^{(n-4) / 2} b>C(n)$ when $n \geq 5$. Noting that a bounded sequence in $H^{1}$ of solutions of (1) is a Palais-Smale sequence for $I$, according to what we proved above, it suffices to prove that if $\left(u_{\alpha}\right)_{\alpha}$ is a sequence of solutions of (1), then $\left(u_{\alpha}\right)_{\alpha}$ is bounded in $H^{1}$ when $n \geq 5$ and when $n=4$ and $b \gg 1$. By the Palais-Smale property we would indeed get that, up to passing to a subsequence, $\left(u_{\alpha}\right)_{\alpha}$ converges in $H^{1}$, and by standard elliptic theory, this actually implies that the sequence converges in $C^{2}$. Now, we multiply the equation by $u_{\alpha}$ and integrate over $M$, yielding

$$
a \int_{M}\left(\left|\nabla u_{\alpha}\right|^{2}+\frac{h}{a} u_{\alpha}^{2}\right) d v_{g}+b\left(\int_{M}\left|\nabla u_{\alpha}\right|^{2} d v_{g}\right)^{2}=\int_{M}\left|u_{\alpha}\right|^{2^{\star}} d v_{g}
$$

for all $\alpha$. We clearly get from (6) and (22) that $\left(u_{\alpha}\right)_{\alpha}$ is bounded in $H^{1}$ if the sequence $\left(\bar{u}_{\alpha}\right)_{\alpha}$ is bounded (and $b \gg 1$ when $n=4$ ). We may thus assume that $\bar{u}_{\alpha} \rightarrow+\infty$ as $\alpha \rightarrow+\infty$. By (6) and (22), we get that (8) holds. Writing (9), we then get that (12) holds and also that

$$
\int_{M}\left|u_{\alpha}\right| d v_{g}=\left|\bar{u}_{\alpha}\right|\left(1+C_{\alpha}\right)
$$

where $C_{\alpha}=O\left(\frac{1}{b}\right)$ if $n=4$, and $C_{\alpha}=o(1)$ if $n \geq 5$. Integrating the equation,

$$
\int_{M} h u_{\alpha} d v_{g}=\int_{M}\left|u_{\alpha}\right|^{2^{\star}-2} u_{\alpha} d v_{g}
$$

The contradiction follows from (12), (23), and (24). This proves the above claim that $\left(u_{\alpha}\right)_{\alpha}$ is bounded in $H^{1}$. This also proves that the set of solutions of (1) is compact in the $C^{2}$-topology.

At this point we define a mountain-pass solution of (1) as a solution which we obtain from $I$ by the use of the mountain-pass lemma. We easily get from Theorem 1 that the following existence result holds true. 
Proposition 2. Suppose that $(M, g)$ is a closed Riemannian manifold of dimension $n \geq 4$, that $a$ and $b$ are positive real numbers, and that $h \in C^{1}(M, \mathbb{R})$ is such that $\Delta_{g}+h / a$ is positive. Assume that $b \gg 1$ when $n=4$, and that $a^{(n-4) / 2} b>C(n)$ when $n \geq 5$, where $C(n)$ is as in (3). Then, (1) possesses a nontrivial mountain-pass solution.

Proof of Proposition 2. Let $u_{0} \equiv 1$. Then, $I$ is $C^{1}, I(0)=0, I\left(T u_{0}\right)<0$ for $T \gg 1$, and by the coercivity of $\Delta_{g}+h / a$, there exist $C_{1}, C_{2}>0$ such that $I(u) \geq C_{1}\|u\|_{H^{1}}^{2}-C_{2}\|u\|_{H^{1}}^{2^{\star}}$ for all $u$. Then, we can apply the mountain-pass lemma of Ambrosetti and Rabinowitz [1973] and we get that there exists a sequence $\left(u_{\alpha}\right)_{\alpha}$ in $H^{1}$ such that $I\left(u_{\alpha}\right)=c+o(1)$ and $I^{\prime}\left(u_{\alpha}\right) .(\psi)=o\left(\|\psi\|_{H^{1}}\right)$ for all $\psi \in H^{1}$, where

$$
c=\inf _{\gamma \in \Gamma} \sup _{u \in \gamma} I(u),
$$

and $\Gamma$ is the set of continuous paths from 0 to $T u_{0}$. Obviously, $c>0$. By Theorem 1 , up to passing to a subsequence, $\left(u_{\alpha}\right)_{\alpha}$ converges in $H^{1}$. Let $u_{\infty}$ be the limit in $H^{1}$ of the sequence $u_{\alpha}$. Then $I\left(u_{\infty}\right)=c, u_{\infty} \not \equiv 0$, and by passing to the limit in the equation $I^{\prime}\left(u_{\alpha}\right) .(\varphi)=o(1)$ for all $\varphi \in H^{1}$, we get that $u_{\infty}$ solves (1).

It is easily seen that the mountain-pass solution $u_{\infty}$ obtained in Proposition 2 has a nice ground-state structure when $n=4$. We define the Nehari manifold $\mathcal{N}$ attached to $I$ by

$$
\mathcal{N}=\left\{u \in H^{1} \backslash\{0\} \mid I^{\prime}(u) .(u)=0\right\} .
$$

The following 4-dimensional ground-state characterization of the solution obtained in Proposition 2 holds true.

Proposition 3. Suppose that $(M, g)$ is a closed 4-dimensional Riemannian manifold, that $a$ and $b$ are positive real numbers, and that $h \in C^{1}(M, \mathbb{R})$ is such that $\Delta_{g}+h / a$ is positive. Assume that $b \gg 1$. Then, the mountain-pass solution $u_{\infty}$ obtained in Proposition 2 has a ground-state structure given by

$$
I\left(u_{\infty}\right)=\inf _{u \in \mathcal{N}} I(u),
$$

where $\mathcal{N}$ is the Nehari manifold attached to I given by (25).

Proof of Proposition 3. We obviously have that $u_{\infty} \in \mathcal{N}$, and thus there holds that $I\left(u_{\infty}\right) \geq \inf _{u \in \mathcal{N}} I(u)$. Given $\tilde{u} \in H^{1} \backslash\{0\}$, we define the mountain-pass energy level $c_{\tilde{u}}$ by

$$
c_{\tilde{u}}=\inf _{\gamma \in \Gamma_{\tilde{u}}} \sup _{u \in \gamma} I(u),
$$

where $\Gamma_{\tilde{u}}$ is the set of continuous paths from 0 to $\tilde{u}$. Let $u_{0} \equiv 1$ be as in the proof of Proposition 2. Let $T_{0} \gg 1$ be such that $I\left(T_{0} u_{0}\right)<0$. By construction (see the proof 
of Proposition 2), it holds that $I\left(u_{\infty}\right)=c_{T_{0} u_{0}}$. Let $u \in \mathcal{N}$. Then $I(u)=I(|u|)$,

$$
a \int_{M}\left(|\nabla u|^{2}+\frac{h}{a} u^{2}\right) d v_{g}+b\left(\int_{M}|\nabla u|^{2} d v_{g}\right)^{2}=\int_{M} u^{4} d v_{g}
$$

and for $t \geq 0$,

$$
\begin{aligned}
I(t|u|) & =\frac{a t^{2}}{2} \int_{M}\left(|\nabla u|^{2}+\frac{h}{a} u^{2}\right) d v_{g}+\frac{b t^{4}}{4}\left(\int_{M}|\nabla u|^{2} d v_{g}\right)^{2}-\frac{t^{4}}{4} \int_{M} u^{4} d v_{g} \\
& =\frac{a t^{2}\left(2-t^{2}\right)}{4} \int_{M}\left(|\nabla u|^{2}+\frac{h}{a} u^{2}\right) d v_{g} .
\end{aligned}
$$

In particular, $I\left(T_{1}|u|\right)<0$ for $T_{1}>\sqrt{2}$. Let $u_{1}=|u|$ and $T_{1} \gg 1$. It is easily checked (since $u_{0}$ is constant) that

$$
I\left(t T_{1} u_{1}+(1-t) T_{0} u_{0}\right) \leq t^{2} I\left(T_{1} u_{1}\right)-\frac{(1-t)^{2} T_{0}^{2} u_{0}^{2} V_{g}}{4}<0
$$

for all $0 \leq t \leq 1$, where $V_{g}$ is the volume of $(M, g)$. In particular, $c_{T_{0} u_{0}}=c_{T_{1} u_{1}}$ since $T_{0} u_{0}$ and $T_{1} u_{1}$ can be connected by a continuous path along which $I$ is everywhere negative. So,

$$
c_{T_{0} u_{0}} \leq \sup _{0 \leq t \leq T_{1}} I\left(t u_{1}\right)
$$

By (27) we see that $t \rightarrow I\left(t u_{1}\right)$ is maximal at $t=1$, and thus $c_{T_{0} u_{0}} \leq I(u)$ by (28). This proves that $I\left(u_{\infty}\right) \leq I(u)$ for all $u \in \mathcal{N}$, and thus that (26) holds.

Balancing Proposition 2 we prove that the following uniqueness result, in the sense of Brézis and Li [2006], holds.

Proposition 4. Suppose that $(M, g)$ is a closed Riemannian manifold of dimension $n \geq 4$ and that $h$ is a positive constant. Let $\varepsilon_{0}>0$ arbitrary. For $a, b \gg 1$ when $n=4$, and $a \gg 1, b \geq \varepsilon_{0}$ when $n \geq 5$, the sole nontrivial pair of solutions of (1) is the pair $(-u, u)$ of constant solutions, where $u=h^{(n-2) / 4}$.

Proof of Proposition 4. Let $\varepsilon_{0}>0$ be given arbitrarily small. We prove the result by contradiction. We assume that there exist sequences $\left(a_{\alpha}\right)_{\alpha},\left(b_{\alpha}\right)_{\alpha}$ of positive real numbers, and a sequence $\left(u_{\alpha}\right)_{\alpha}$ of nonconstant solutions of

$$
\left(a_{\alpha}+b_{\alpha} \int_{M}\left|\nabla u_{\alpha}\right|^{2} d v_{g}\right) \Delta_{g} u_{\alpha}+h u_{\alpha}=\left|u_{\alpha}\right|^{2^{\star}-2} u_{\alpha}
$$

for all $\alpha$ such that $a_{\alpha} \rightarrow+\infty$ and $b_{\alpha} \rightarrow+\infty$ as $\alpha \rightarrow+\infty$ when $n=4$, and such that $a_{\alpha} \rightarrow+\infty$ as $\alpha \rightarrow+\infty$ and $b_{\alpha} \geq \varepsilon_{0}$ for all $\alpha$ when $n \geq 5$. As in the proof 
of Theorem 1, this implies that $\left\|u_{\alpha}\right\|_{H^{1}}=O(1)$. Suppose that $K_{\alpha}$ is as in (14), $h_{\alpha}=K_{\alpha}^{-1} h$, and $v_{\alpha}$ is as in (15). Then,

$$
\Delta_{g} v_{\alpha}+h_{\alpha} v_{\alpha}=\left|v_{\alpha}\right|^{2^{\star}-2} v_{\alpha}
$$

and $K_{\alpha} \rightarrow+\infty$ since $a_{\alpha} \rightarrow+\infty$ as $\alpha \rightarrow+\infty$. Then, by elliptic regularity, $v_{\alpha} \rightarrow 0$ in $C^{0}$. Multiplying (30) by $v_{\alpha}-\bar{v}_{\alpha}$, and integrating over $M$,

$$
\begin{aligned}
\lambda_{1} \int_{M}\left(v_{\alpha}-\bar{v}_{\alpha}\right)^{2} d v_{g} & \leq \int_{M}\left(v_{\alpha}-\bar{v}_{\alpha}\right)\left(\left|v_{\alpha}\right|^{2^{\star}-2} v_{\alpha}-\left|\bar{v}_{\alpha}\right|^{2^{\star}-2} \bar{v}_{\alpha}\right) d v_{g} \\
& \leq C\left\|v_{\alpha}\right\|_{L^{\star}}^{2^{\star}-2} \int_{M}\left(v_{\alpha}-\bar{v}_{\alpha}\right)^{2} d v_{g}
\end{aligned}
$$

for all $\alpha$, where $C>0$ is independent of $\alpha$, and $\lambda_{1}>0$ is the first nontrivial eigenvalue of $\Delta_{g}$. Since $v_{\alpha} \rightarrow 0$ in $C^{0}$, (31) implies that $v_{\alpha}=\bar{v}_{\alpha}$, and we get a contradiction.

\section{Acknowledgements}

The author would like to thank Pierre-Damien Thizy for helpful and valuable comments on the manuscript, and for pointing out the very nice Proposition 4.

\section{References}

[Ambrosetti and Rabinowitz 1973] A. Ambrosetti and P. H. Rabinowitz, "Dual variational methods in critical point theory and applications", J. Functional Analysis 14 (1973), 349-381. MR 51 \#6412 Zbl 0273.49063

[Brezis and Li 2006] H. Brezis and Y. Li, "Some nonlinear elliptic equations have only constant solutions", J. Partial Differential Equations 19:3 (2006), 208-217. MR 2007f:35076 Zbl 1174.35368

[Druet et al. 2004] O. Druet, E. Hebey, and F. Robert, Blow-up theory for elliptic PDEs in Riemannian geometry, Mathematical Notes 45, Princeton University Press, 2004. MR 2005g:53058 Zbl 1059.58017

[Figueiredo 2013] G. M. Figueiredo, "Existence of a positive solution for a Kirchhoff problem type with critical growth via truncation argument", J. Math. Anal. Appl. 401:2 (2013), 706-713. MR 3018020 Zbl 1307.35110

[Figueiredo and Santos 2012] G. M. Figueiredo and J. R. Santos, Jr., "Multiplicity of solutions for a Kirchhoff equation with subcritical or critical growth", Differential Integral Equations 25:9-10 (2012), 853-868. MR 2985683 Zbl 1274.35087

[Figueiredo et al. 2014] G. M. Figueiredo, N. Ikoma, and J. R. Santos, Jr., "Existence and concentration result for the Kirchhoff type equations with general nonlinearities", Arch. Ration. Mech. Anal. 213:3 (2014), 931-979. MR 3218834 Zbl 1302.35356

[He and Zou 2012] X. He and W. Zou, "Existence and concentration behavior of positive solutions for a Kirchhoff equation in $\mathbb{R}^{3}$ ", J. Differential Equations 252:2 (2012), 1813-1834. MR 2853562 Zbl 1235.35093

[Hebey 2014] E. Hebey, Compactness and stability for nonlinear elliptic equations, European Mathematical Society, Zürich, 2014. MR 3235821 Zbl 1305.58001 
[Hebey and Thizy 2015a] E. Hebey and P. D. Thizy, "Stationary Kirchhoff systems in closed 3dimensional manifolds", Calc. Var. Partial Differential Equations (online publication April 2015).

[Hebey and Thizy 2015b] E. Hebey and P. D. Thizy, "Stationary Kirchhoff systems in closed high dimensional manifolds", Commun. Contemp. Math. (online publication April 2015).

[Kirchhoff 1883] G. Kirchhoff, Vorlesungen über Mechanik, 3rd ed., Vorlesungen über Mathematische Physik 1, Teubner, Leipzig, 1883. JFM 09.0597.01

[Lions 1978] J.-L. Lions, "On some questions in boundary value problems of mathematical physics", pp. 284-346 in Contemporary developments in continuum mechanics and partial differential equations (Rio de Janeiro, 1977), edited by G. M. de la Penha and L. A. J. Medeiros, North-Holland Math. Stud. 30, North-Holland, Amsterdam, 1978. MR 82b:35020 Zbl 0404.35002

[Struwe 1984] M. Struwe, "A global compactness result for elliptic boundary value problems involving limiting nonlinearities”, Math. Z. 187:4 (1984), 511-517. MR 86k:35046 Zbl 0535.35025

[Vétois 2007] J. Vétois, "Multiple solutions for nonlinear elliptic equations on compact Riemannian manifolds”, Internat. J. Math. 18:9 (2007), 1071-1111. MR 2009f:53051 Zbl 1148.35026

Received April 17, 2015. Revised May 3, 2015.

EMMANUEl HeBEy

DÉPARTEMENT DE MATHÉMATIQUES

Université DE CERGY-Pontoise

2 AVENUE Adolphe ChaUVIN

95302 CERGY-PONTOISE

FRANCE

Emmanuel.Hebey@math.u-cergy.fr 


\title{
PACIFIC JOURNAL OF MATHEMATICS
}

\author{
msp.org/pjm
}

Founded in 1951 by E. F. Beckenbach (1906-1982) and F. Wolf (1904-1989)

\section{EDITORS}

Don Blasius (Managing Editor)

Department of Mathematics

University of California

Los Angeles, CA 90095-1555

blasius@math.ucla.edu

\author{
Paul Balmer \\ Department of Mathematics \\ University of California \\ Los Angeles, CA 90095-1555 \\ balmer@math.ucla.edu \\ Robert Finn \\ Department of Mathematics \\ Stanford University \\ Stanford, CA 94305-2125 \\ finn@math.stanford.edu \\ Sorin Popa \\ Department of Mathematics \\ University of California \\ Los Angeles, CA 90095-1555 \\ popa@math.ucla.edu
}

\author{
Vyjayanthi Chari \\ Department of Mathematics \\ University of California \\ Riverside, CA 92521-0135 \\ chari@math.ucr.edu \\ Kefeng Liu \\ Department of Mathematics \\ University of California \\ Los Angeles, CA 90095-1555 \\ liu@math.ucla.edu \\ Jie Qing \\ Department of Mathematics \\ University of California \\ Santa Cruz, CA 95064 \\ qing@ cats.ucsc.edu
}

\section{PRODUCTION}

Silvio Levy, Scientific Editor, production@msp.org

\section{SUPPORTING INSTITUTIONS}

ACADEMIA SINICA, TAIPEI

CALIFORNIA INST. OF TECHNOLOGY

INST. DE MATEMÁTICA PURA E APLICADA

KEIO UNIVERSITY

MATH. SCIENCES RESEARCH INSTITUTE

NEW MEXICO STATE UNIV.

OREGON STATE UNIV.

\author{
STANFORD UNIVERSITY \\ UNIV. OF BRITISH COLUMBIA \\ UNIV. OF CALIFORNIA, BERKELEY \\ UNIV. OF CALIFORNIA, DAVIS \\ UNIV. OF CALIFORNIA, LOS ANGELES \\ UNIV. OF CALIFORNIA, RIVERSIDE \\ UNIV. OF CALIFORNIA, SAN DIEGO \\ UNIV. OF CALIF., SANTA BARBARA
}

\author{
Daryl Cooper \\ Department of Mathematics \\ University of California \\ Santa Barbara, CA 93106-3080 \\ cooper@math.ucsb.edu \\ Jiang-Hua Lu \\ Department of Mathematics \\ The University of Hong Kong \\ Pokfulam Rd., Hong Kong \\ jhlu@maths.hku.hk \\ Paul Yang \\ Department of Mathematics \\ Princeton University \\ Princeton NJ 08544-1000 \\ yang@math.princeton.edu
}

These supporting institutions contribute to the cost of publication of this Journal, but they are not owners or publishers and have no responsibility for its contents or policies.

See inside back cover or msp.org/pjm for submission instructions.

The subscription price for 2016 is US $\$ 440 /$ year for the electronic version, and $\$ 600 /$ year for print and electronic.

Subscriptions, requests for back issues and changes of subscribers address should be sent to Pacific Journal of Mathematics, P.O. Box 4163, Berkeley, CA 94704-0163, U.S.A. The Pacific Journal of Mathematics is indexed by Mathematical Reviews, Zentralblatt MATH, PASCAL CNRS Index, Referativnyi Zhurnal, Current Mathematical Publications and Web of Knowledge (Science Citation Index).

The Pacific Journal of Mathematics (ISSN 0030-8730) at the University of California, c/o Department of Mathematics, 798 Evans Hall \#3840, Berkeley, CA 94720-3840, is published twelve times a year. Periodical rate postage paid at Berkeley, CA 94704, and additional mailing offices. POSTMASTER: send address changes to Pacific Journal of Mathematics, P.O. Box 4163, Berkeley, CA 94704-0163.

PJM peer review and production are managed by EditFLOW ${ }^{\circledR}$ from Mathematical Sciences Publishers.

\section{PUBLISHED BY}

\section{mathematical sciences publishers \\ nonprofit scientific publishing}

http://msp.org/

(C) 2016 Mathematical Sciences Publishers 


\section{PACIFIC JOURNAL OF MATHEMATICS}

Volume $280 \quad$ No. $1 \quad$ January 2016

Stable capillary hypersurfaces in a wedge

JAIGYOUNG CHOE and MIYUKI KoISO

The Chern-Simons invariants for the double of a compression body

DAVID L. DUNCAN

Compactness and the Palais-Smale property for critical Kirchhoff equations in

closed manifolds

EMMANUEL HeBEy

On the equivalence of the definitions of volume of representations

SUNGWOON KIM

Strongly positive representations of even GSpin groups

YEANSU KIM

An Orlik-Raymond type classification of simply connected 6-dimensional torus manifolds with vanishing odd-degree cohomology

\section{SHINTARÔ KUROKI}

Solutions with large number of peaks for the supercritical Hénon equation

Zhongyuan LiU and ShuangJie Peng

Effective divisors on the projective line having small diagonals and small heights and 141 their application to adelic dynamics

YÛSUKE OKUYAMA

Computing higher Frobenius-Schur indicators in fusion categories constructed from inclusions of finite groups

\section{Peter Schauenburg}

Chordal generators and the hydrodynamic normalization for the unit ball

SEBASTIAN SCHLEISSINGER

On a question of A. Balog

ILYA D. SHKREDOV

Uniqueness result on nonnegative solutions of a large class of differential inequalities 241 on Riemannian manifolds

YUHUA SUN

Correction to "Closed orbits of a charge in a weakly exact magnetic field"

WILL J. MERRY 